\title{
What Can the Accretion Induced Collapse of White Dwarfs Really Explain?
}

\author{
Chris L. Fryer \\ Lick Observatory, University of California at Santa Cruz, Santa Cruz, CA 95064 \\ Willy Benz \\ Physikalisches Institut, Universitaet Bern, Sidlerstrasse 5, CH-3012 Bern Switzerland \\ Marc Herant \\ Washington University School of Medicine, Box 8107, 660 S. Euclid, St. Louis, MO 63110 \\ Stirling A. Colgate \\ Theoretical Division, T-6, MS B288, Los Alamos National Laboratory, Los Alamos, NM 87545
}

\begin{abstract}
The accretion induced collapse (AIC) of a white dwarf into a neutron star has been invoked to explain gamma-ray bursts, Type Ia supernovae, and a number of problematic neutron star populations and specific binary systems. The ejecta from this collapse has also been claimed as a source of r-process nucleosynthesis. So far, most AIC studies have focussed on determining the event rates from binary evolution models and less attention has been directed toward understanding the collapse itself. However, the collapse of a white dwarf into a neutron star is followed by the ejection of rare neutron-rich isotopes. The observed abundance of these chemical elements may set a more reliable limit on the rate at which AICs have taken place over the history of the galaxy.

In this paper, we present a thorough study of the collapse of a massive white dwarf in 1and 2-dimensions and determine the amount and composition of the ejected material. We discuss the importance of the input physics (equation of state, neutrino transport, rotation) in determining these quantities. These simulations affirm that AICs are too baryon rich to produce gamm-ray bursts and do not eject enough nickel to explain Type Ia supernovae (with the possible exception of a small subclass of extremely low-luminosity Type Ias). Although nucleosynthesis constraints limit the number of neutron stars formed via AICs to $\lesssim 0.1 \%$ of the total galactic neutron star population, AICs remain a viable scenario for forming systems of neutron stars which are difficult to explain with Type II core-collapse supernovae.
\end{abstract}

Subject headings: stars: neutron - stars: white dwarfs - pulsars

\section{Introduction}

White dwarfs accreting up to the Chandrasekhar limit follow one of two paths. Either the densities and temperatures become sufficiently high to ignite explosive nuclear burning, disrupting the white dwarf in what is now considered the favored Type Ia explosion mechanism (see Woosley \& Weaver 1986 for a summary), or electron capture reduces central temperatures and pressures and instead drives a collapse of the white dwarf. This collapse leads to the formation of a neutron star and is similar to the core collapse of massive stars, the mechanism behind Type II supernovae. Just as the core collapse of massive stars ejects 
material, one might also expect material to be ejected from the accretion induced collapse (AIC) of white dwarfs.

Assuming some mass ejection occurs during collapse, AICs have been proposed as an alternate mechanism for Type Ia supernova (Colgate, Petschek, \& Kriese 1980) and as a source for gamma-ray bursts (Paczynski 1986, Goodman 1986; Goodman, Dar, \& Nussinov 1987; Paczynski 1990; Dar et al. 1992). Neutron stars formed through AICs have been used to explain a variety of troublesome neutron star systems (see Canal, Isern, \& Labay 1990 for a review). AICs have been proposed as an alternate channel to form neutron stars in globular clusters and in the galactic disk, the most common being millisecond pulsars (Bailyn \& Grindlay 1988; Bailyn \& Grindlay 1990; Kulkarni, Narayan, \& Romani 1990; Ray \& Kluzniak 1990; Ruderman 1991; Chen \& Ruderman 1993; Chen \& Leonard 1993). AICs have also been invoked in several X-ray binary formation scenarios (Canal et al. 1990, van den Heuvel 1984) and as a formation mechanism for specific cases of close neutron star binaries (Ergma 1993).

The role AICs play to produce these objects depends upon their rate. Super-soft X-ray sources are possible candidates of white dwarfs accreting up to the Chandrasekhar limit (Li \& van den Heuvel 1997). However, whether or not the white dwarf will form a Type Ia supernova in a thermonuclear explosion or collapse into a neutron star in an AIC depends sensitively upon the initial white dwarf mass, white dwarf composition, and the accretion rate onto the white dwarf (Nomoto 1982, 1984; Nomoto \& Kondo 1991). Although current observations verify that likely progenitors for AICs do exist, they do not place strong quantitative constraints on the event rate of these collapses. Similarly, uncertainties in binary evolution and white dwarf formation make it difficult to predict any definitive AIC event rate from population synthesis calculations (Yungelson \& Livio 1998).

By simulating the collapse of white dwarfs, and their subsequent explosions, we can constrain the viability of AICs as gamma-ray bursts and Type Ia supernovae mechanisms. In addition, we can also use the nucleosynthetic yield from the ejecta of AICs to place limits on the event rate. The ejecta of AICs is neutron rich and leads to the production of many anomalous neutron-rich isotopes (e.g. ${ }^{62} \mathrm{Ni},{ }^{66} \mathrm{Zn},{ }^{68} \mathrm{Zn}$, ${ }^{87} \mathrm{Rb}$, and ${ }^{88} \mathrm{Sr}$ ) which pollute the interstellar medium. By comparing the observed abundance of these elements with the amount ejected per AIC event, we can place constraints on the allowable rate of AICs in the galaxy (Woosley \& Baron 1987, hereafter WB87).

Previous work on AICs has identified three possible mass-ejection mechanisms: the prompt mechanism driven by the bounce of the collapsing white dwarf as the core reaches nuclear densities, the "delayedneutrino" mechanism which occurs shortly after the bounce-shock stalls (20-200 ms) and is driven by neutrino absorption, and the neutrino wind mechanism which is a relatively stable mass-loss occurring over the relatively long cooling timescales (1-2s) of the proto-neutron star. Baron et al. (1987), Mayle \& Wilson (1988) and Woosley \& Baron (1992) all found that the bounce shock stalls due to the energy losses from neutrino emission and dissociation and the prompt mechanism fails to drive an explosion. Mayle \& Wilson's simulations of the collapse of massive star $\left(8-10 M_{\odot}\right)$ OMgNe cores, which have similar structures to most AIC progenitor models, led to explosions on short timescales $(\sim 200 \mathrm{~ms})$ via the delayed-neutrino mechanism with $0.042 M_{\odot}$ ejected. Simulations by Hillebrandt, Nomoto, \& Wolff (1984) of the collapse of $\mathrm{OMgNe}$ white dwarfs ejected as much as $\sim 0.1-0.2 M_{\odot}$ with explosions which developed even sooner (20-30 ms). These explosions occur so quickly because the collapsing white dwarf does not have a huge infalling mantle that provides a ram pressure containing, at least temporarily, the explosion. WB92, however, found that no such explosion resulted from the collapse of a CO white dwarf. The only mass-loss $\left(\sim 0.01 M_{\odot}\right)$ occurred at late times through the proto-neutron star's neutrino-driven wind. 
In both the delayed-neutrino explosion mechanism and the neutrino-driven wind, the material ejected is likely to be neutron rich. Shortly after the inception of the neutrino-driven supernova mechanism, it was realized (Arnett \& Truran 1970) that the densities and temperatures near the neutrinosphere are sufficiently high to force this material to deleptonize via the emission of electron neutrinos. This material is ejected with extremely low electron fractions $\left(0.35<Y_{e}<0.45\right)$. In Type II supernovae simulations, the assumption is that either a longer delay in the supernova explosion (due to the ram-pressure of the infalling material) causes most of this material to remain part of the neutron star, or that the neutron-rich material falls back on the neutron star. The fallback is driven by the reverse shock that is created as the supernova shock wave traverses the envelope of the massive star. As we shall show in this paper, neither of these arguments can possibly hold for AICs and we can not easily explain away the low $Y_{e}$ ejecta from the delayed neutrino mechanism.

Neutrino emission is not the only way to lower the electron fraction of matter. The electron fraction neutrino-wind driven ejecta is set by the relative absorption of electron neutrinos and anti-neutrinos (Qian et al. 1993). Qian et al. (1993) have shown that since the neutrinosphere of the electron anti-neutrinos is deeper within the proto-neutron star crust, the anti-neutrinos are more energetic than the electron neutrinos. Since the neutrino cross-section is proportional to the square of the neutrino energy, given the same flux of neutrino/anti-neutrinos, the wind driven material is likely to absorb more anti-neutrinos and it becomes neutron rich.

In this paper, we give results from a series of AIC simulations using the CO white dwarf progenitor from the WB92 model to determine the amount and composition of the ejecta. To measure the reliability of our results, we vary a number of parameters such as the details of the neutrino physics, the equation of state, and the initial rotation of the white dwarf. In particular, we are able to understand the difference between the WB92 results and the other groups. We discuss the models in detail in $\S 2$. A summary of these results and their implications are given in $\S 3$.

\section{Models and Results}

Table 1 summarizes the entire set (60 in total) of simulations we have performed. The different simulations were run to test the sensitivity of the results to changes in the neutrino physics (both source and transport columns 3 and 4 of Table 1), the inclusion of relativistic effects, (runs 6, 11, and 17), the

choice of the equation of state (EOS) (column 2), multi-dimensional effects (run 2) and initial rotation rate of the white dwarf (run 3).

Except for changes in the equation of state for dense matter, most of these parameter variations lead to relatively small changes in the results (factors of 2 in the mass ejected). Changes in the equation of state explain the differences between the previous simulations (Hillebrandt, Nomoto \& Wolff 1984 and Mayle \& Wilson 1988 versus Woosley \& Baron 1992). We include calculations using both the equation of state of Swesty \& Lattimer (1992) and that of Baron, Cooperstein, \& Kahana (1985). The large differences can be appreciated by comparing the mass-point trajectories over the course of the simulation (Figs 1,2). In this section we discuss the specific variations in our simulations and their effects on the results.

To calculate the upper limit of the event rate of AICs, we must estimate the nucleosynthetic yield of the neutron rich ejecta. Hartmann, Woosley, and El Eid (1985) estimate that there must be less than $10^{-5} M_{\odot}$ of $Y_{e}<0.4$ material ejected per supernova to avoid anomalous abundances of particular isotopes (e.g. ${ }^{62} \mathrm{Ni},{ }^{66} \mathrm{Zn},{ }^{68} \mathrm{Zn},{ }^{87} \mathrm{Rb}$, and ${ }^{88} \mathrm{Sr}$ ). Using the value of $0.02 M_{\odot}$ of material with less than $Y_{e}<0.4$ 
ejected per AIC events (see Table 1) and assuming a supernova rate of two per century for the Galaxy we find that the upper limit for rate of AICs must be $\left(2 / 100 \mathrm{y}^{-1}\right)\left(10^{-5} M_{\odot}\right) /\left(0.02 M_{\odot}\right)=10^{-5} \mathrm{y}^{-1}$. Note that we have assumed AICs to be the only source for material with such a low $Y_{e}$. Should there be another source of these neutron rich isotopes, the allowed AIC rate will be correspondingly smaller. A similar constraint can be calculated by using the material ejected with $0.45<Y_{e}<0.40$ rather than with $Y_{e}<0.4$. Following the method of WB92 for $0.02 M_{\odot}$ of ejected material, the upper limit for the rate of AICs becomes $\left(1 M_{\odot} / 170,000\right)\left(1 / 0.02 M_{\odot}^{-1}\right)(1 / 0.13)\left(2 / 100 y^{-1}\right)=4.5 \times 10^{-5} \mathrm{y}^{-1}$. In table 1 , we list the upper limit of the event rate for each simulation. In parentheses, we list the same upper limit if the electron fraction of the ejecta is $30 \%$ higher than the predictions in our simulations. We note that much of the ejecta has a very low electron fraction $\left(Y_{e}<0.3\right)$ and large errors in the estimated electron fraction would be required to change the upper limit of the AIC event rate by more than an order of magnitude.

\subsection{Numerical Methods}

The internal structure of the initial white dwarf is taken from the progenitor used by WB92. This model is then mapped into our one- and/or two-dimensional codes and run for $0.2 \mathrm{~s}$. The one-dimensional simulations were performed using the code developed and tested in previous work (Benz 1991; Herant et al. 1994; Fryer, Benz \& Herant 1996) with $\sim 110$ zones where the highest resolution was constructed near the mass cut. This code does not include any form of convection modeling (mixing length or other). The two-dimensional simulations were performed using the Smooth Particle Hydrodynamics (SPH) code discussed in Herant et al. (1994) with typically $\sim 8000$ particles. We model a $180^{\circ}$ wedge assuming cylindrical symmetry about the angular momentum axis and also run these models for $0.2 \mathrm{~s}$. As described in Herant et al. (1994), both codes use the same implementations of neutrino physics and transport, equations of state, etc. Thus, we can use the two dimensional simulations to compare the effects of convection and rotation.

For some of the one-dimensional simulations, we include general relativistic effects using the formalism developed by van Riper (1997). For our late-time simulations, we have added a cell-splitting routine which allows us to follow the evolution of the explosion long after the collapse (we have run select simulations to $0.5 \mathrm{~s}$ ). We only use the cell-splitting routine at late times, after the explosion has occurred. When the cell size becomes a sizable fraction (0.3) of its radius, we divide the cell in half and reduce the energy (and hence pressures) of the inner cell by $5 \%$ to allow the forces at the boundaries to remain roughly equal.

\section{2. $\quad$ Effects of Neutrino Physics}

To illustrate the importance of neutrinos on the composition of the ejecta, we compare the results of a simulation which includes the effects of neutrino physics (Fig. 1 - run 1) and a simulation with no neutrino emission or absorption (Fig. 3 - run 24). By comparing the mass-point trajectories between these two simulations, we note that without the cooling effects of neutrino emission, the bounce shock does not stall and an explosion develops (a "prompt" explosion). For the simulation which includes the effects of neutrino physics, neutrino emission from the shocked material (along with dissociation) stalls the shock. The neutrino emission from the shocked material and the new material falling onto the shocked region serves to deleptonize the material. Using the Swesty-Lattimer (1992) equation of state, we find that neutrino heating is able to revive the explosion, ejecting $\sim 0.1 M_{\odot}$ of material. The neutrino emission and later absorption 
sets the electron fraction of the material. However, very quickly, the ejecta is thrown sufficiently far where adiabatic cooling causes recombination which lowers the free proton and free neutron fraction. The neutrino opacity of this material drops, and the electron fraction is effectively "frozen-out". In all cases where the delayed-neutrino mechanism is the dominant mass ejector, we follow our simulations until this occurs.

Including neutrino physics in the simulations involves two difficulties: the determination and numerical representation of the processes that emit or absorb neutrinos and the subsequent transport of these neutrinos through matter. Our neutrino processes are described in Herant et al. (1994) and include many of the possible emission and absorption rates for the standard three neutrino species (electron neutrino, electron antineutrino, and the entire set of $\mu$ and $\tau$ neutrinos and antineutrinos).

In many Type II supernova simulations, it is often assumed that the neutrinos are emitted from ultra-relativistic electrons $\left(T \gg \frac{1}{2} \mathrm{MeV}\right)$. This is not always necessarily a good approximation. An analytical approximation of the electron/positron neutrino emission rates and luminosity has been derived by Takahashi, El Eid \&Hillebrandt (1977):

$$
\begin{aligned}
& \lambda_{\substack{e^{-p} \\
e^{+} n}}=C_{2} \beta^{-5}\left[F_{4}( \pm \eta) \pm(2-2 \Delta) F_{3}( \pm \eta) \beta+\left(\frac{1-8 \Delta+2 \Delta^{2}}{2}\right) F_{2}( \pm \eta) \beta^{2}\right. \\
& \left. \pm\left(2 \Delta^{2}-\Delta\right) F_{1}( \pm \eta) \beta^{3}+\left(\frac{4 \Delta^{2}-1}{8}\right) F_{0}( \pm \eta) \beta^{4}\right]
\end{aligned}
$$

and

$$
\begin{aligned}
& L_{\bar{\nu}}=C_{3} \beta^{-6}\left[F_{5}( \pm \eta) \pm(2-3 \Delta) F_{4}( \pm \eta) \beta+\left(\frac{1-12 \Delta+6 \Delta^{2}}{2}\right) F_{3}( \pm \eta) \beta^{2}\right. \\
& \pm\left(\frac{-3 \Delta \pm 12 \Delta^{2}-2 \Delta^{3}}{2}\right) F_{2}( \pm \eta) \beta^{3}+\left(\frac{-1+12 \Delta^{2}-16 \Delta^{3}}{8}\right) F_{1}( \pm \eta) \beta^{4} \\
& \left. \pm\left(\frac{2+3 \Delta-4 \Delta^{3}}{8}\right) F_{0}( \pm \eta) \beta^{5}\right]
\end{aligned}
$$

where $\lambda_{\substack{e^{-p} \\ e^{+} n}}$ are the transition rates for electron and positron capture, $L_{\bar{\nu}}$ are the electron neutrino and anti-electron neutrino luminosities, $C_{2}=6.15 \times 10^{-4} \mathrm{~s}^{-1}$ per nucleon, $C_{3}=5.04 \times 10^{-10} \mathrm{erg} \mathrm{s}^{-1}$ per nucleon, $\Delta=1.531 \mathrm{MeV}$ is the neutron-hydrogen mass difference and $\beta=\frac{m_{e} c^{2}}{k_{B} T} . F_{n}$ are fermi integrals of order $\mathrm{n}$ and $\eta$ is the degeneracy parameter.

Adopting the ultra-relativistic limit, WB92 have simplified these equations, taking only the first term in each equation. To estimate the importance of this assumption, we have used both the limited and the full equations. In Table 1, the runs using the full equations are identified by the letters TEH while those using the ultra-relativistic assumption are marked by WB. As can be seen from a careful comparison of these simulations, adopting the ultra-relativistic limit changes the amount of neutron rich ejecta (and the corresponding AIC event rate) by less than a factor of 2 .

Despite the low cross-sections for neutrino interactions, the high densities involved in core-collapse scenarios place the neutrinos within the depths of the collapsing star in the diffusion regime. Thus, neutrino transport must include both the diffusion and free-streaming limits of the transport equations. The "standard" approximation to couple these two extremes calls upon the use of a flux-limiter (see for example, Janka 1991). We have incorporated several different flux-limiters (Bowers \& Wilson 1982; Levermore \& Pomraning (1981); Herant et al. 1994) and the properties of their ejecta can be compared in 
column 2 of Table 1. The Bowers-Wilson and Levermore-Pomraning flux limiters seem to bound the more accurate Monte-Carlo calculations by Janka (1991) and can be used to gauge the effect of the flux-limiter on the amount and composition of the ejecta. By comparing these two flux limiters in otherwise identical simulations (e.g. run 8 and 12), we see that the two approximations in the neutrino diffusion lead to only $10 \%$ differences in the mass ejected. The upper limit on the AIC rate with these two flux limiters varies by factors of 2 .

\subsection{Impact of the Equation of State}

Uncertainties surrounding the equation of state for dense matter lead to the largest differences in the ejecta from AICs. We use two such equations of state: the one described in Herant et al. (1994) which couples the nuclear equation of state by Lattimer \& Swesty (1991) to a low density equation of state (Blinnikov, Dunina-Barkovskaya \& Nadyozhin 1996) and a nuclear statistical equilibrium (NSE) scheme (Hix et al. 1994) (hereafter called SL EOS); and the equation of state developed by Baron, Cooperstein, \& Kahana (1985 hereafter called BCK EOS) which covers both low and high density regimes. The BCK EOS is the equation of state used by WB92. For our progenitor, the effects of nuclear burning were minimal. We verified this by running a simulation (run 7) in which we calculate the energy from nuclear burning with a 14 element nuclear network (Benz, Hills, \& Thielemann 1989) rather than assuming nuclear statistical equlibrium. As with the neutrino physics, nuclear burning varies the critical AIC rate by factors of 2 only.

The main results of our simulations using one of the two equations of state are listed in Table 1 (see column 1 - e.g. compare runs 8 and 20). By using the BCK equation of state, we recover the results of WB92. The equation of state dramatically affects the amount and compositon of the ejecta in the simulations. Swesty, Lattimer, \& Myra (1994), in a previous comparison between the two equations of state, report similar findings (the softer BCK equation of state leads to denser, and hotter, cores after bounce). These differences have been discussed by Swesty, Lattimer, \& Myra who argue that, given the standard equation of state parameters for the BCK EOS, the SL EOS is physically more accurate. We have run the SL EOS using two values for the incompressibility of bulk nuclear matter $\left(K_{s}=180,375 \mathrm{MeV}\right)$ and have run a grid of the faster BCK EOS varying the BCK gamma $(1.5<\gamma<3.5)$, the bulk surface coefficient $\left(25<W_{s}<38\right)$, the symmetric bulk compressibility parameter $\left(180 \mathrm{MeV}<K_{0}^{\text {sym }}<375 \mathrm{Mev}\right)$, and an asymmetry parameter $(1.5<x k z<3.5)$. Table 2 gives the results for this grid of simulations in which we use the Levermore-Pomraning flux limiter. Despite the wide range in the physical parameters, the results from the BCK EOS never agree with those from the SL EOS. Clearly, the differences between the two equations of state goes beyond compressibility or asymmetry parameters.

The ratio of the SL EOS pressure to the BCK EOS pressure along an $S=2 \mathrm{k}_{B} /$ nucleon adiabat is plotted in Figure 4. Note that for densities less than $10^{14} \mathrm{~g} \mathrm{~cm}^{-3}$, the pressure of the BCK EOS is 10-20\% greater than that of the SL EOS. Relatively small differences such as these mark the difference between a success or failure of the delayed-neutrino explosion mechanism, which then leads to greater than order-of-magnitude differences in the ejecta!

We have also studied the effects of general relativity on the simulations using the SL equation of state. From Table 1, we can compare the results of simulations with or without general relativity (runs 1,6). The primary effect of general relativity is to cause the material to fall deeper into the potential well resulting in increased neutrino emission/absorption. Just as the equation of state strongly affects the amount and composition of the ejecta, the addition of general relativity leads to variations of over an order of magnitude 
in the upper limit of AIC event rate.

\subsection{Effects of Convection and Rotation}

Two-dimensional simulations of AICs can be used to test both the effects of convection and rotation. Since large entropy gradients do not develop in the one-dimensional simulations, we do not expect convection to cause large differences in the ejecta from AICs. We first use our two-dimensional simulations to verify that convection does not play a major role in the collapse and ejecta of AICs. Although convection is indeed present in the simulations (Fig. 5), the explosion occurs so rapidly $(<100 \mathrm{~ms})$ that it has no time to alter the explosion results. The small differences between the one- and two-dimensional models (compare run 1 and run 2 in Table 1) are probably entirely due to the resolution differences between the two simulations.

The progenitors to AICs accrete not only mass, but angular momentum, as the white dwarf approaches the Chandrasekhar limit. Typical rotation periods for cataclysmic variables range from $200-1200 \mathrm{~s}$ (Liebert 1980) although periods of $\sim 30$ s exist (King \& Lasota 1991). A lower limit on the rotation period is set by the break-up spin period ( $\sim 0.5 \mathrm{~s}$ for solar-mass white dwarfs). In our models, we assume solid-body rotation and conserve each individual particle's angular momentum for the duration of the simulation. We take an extreme case of a white dwarf rotating with a 20 s period prior to collapse (Fig. 5).

For this short rotation period, the ratio of surface rotational velocity over the keplerian velocity of the outer material exceeds 0.1 as the white dwarf collapses. This will certainly alter the flow of the outer material. However, since white dwarfs are probably solid-body rotators, the bulk of the inner material is unaffected by rotation. For example, the ratio of rotational over keplerian velocity drops to 0.01 at the radius which encloses $1.2 M_{\odot}$ (still $87 \%$ of the white dwarf mass). Thus, while the collapse of the outer envelope of a white dwarf is affected by rotation, the core collapse itself is not (compare $\mathrm{T}=50,70 \mathrm{~ms}$ in Fig. 5). Since most of the material exterior to $1.2 M_{\odot}$ is ejected, the net effect of rotation is negligible. Comparing runs 2 and 3 in Table 1, we see that the amount and composition of the ejecta is not altered significantly by the effects of rotation. In addition, there is no preferential ejection of material along either axis.

We do not follow the proto-neutron star as it cools and contracts and the spin period at the end of our simulation (where the hot proto-neutron star's radius is $30-50 \mathrm{~km}$ ) is still $\sim 1 \mathrm{~s}$. Although much of the white dwarf's angular momentum is ejected with the outer $0.2 M_{\odot}$, as the proto-neutron star continues to contract down to $10 \mathrm{~km}$, unless further material is ejected, its spin period will decrease to $10 \mathrm{~ms}$. This

system is likely to continue to accrete from the same companion that caused it to collapse in the first place and this may speed up the neutron star's spin even further, producing millisecond spin periods.

\subsection{Neutrino Wind}

The results in Table 1 do not include any mass loss from neutrino-driven winds. The amount of this ejecta is small when compared to that of our simulations using the SL equation of state (WB92 predict $\sim 0.005 M_{\odot} \mathrm{s}^{-1}$ for the first two seconds). However, for the BCK EOS simulations, neutrino-driven winds dominate the mass loss. Using a cell-adding routine, we follow the fate of an AIC model beyond the delayed neutrino-induced explosion to obtain the wind driven mass loss (run 1). Because we are also modeling the core, we are limited to very small timesteps and are able to follow this phase for only $0.5 \mathrm{~s}$ after bounce. 
We find that during this period of time, an additional $\sim 0.002 M_{\odot}$ peels off the neutron star. Thus, in this limited way, we confirm the results obtained by WB92 that further neutrino driven mass loss is to be expected. Independent of the equation of state or other input physics, it is likely that a neutrino-wind phase exists and that $\sim 0.01 M_{\odot}$ is ejected during this phase. However, the electron fraction of such a wind is very sensitive to the flux and energy of the electron neutrino/anti-neutrinos. We do not list the upper limits for the AIC event rate placed by "wind" ejecta, but prefer to rely upon the ejecta from the delayed-neutrino mechanism which we are better able to test with our simulations.

\section{So What Are AIC Good For?}

Table 1 summarizes the results of our suite of accretion-induced collapse simulations varying the input physics within the range of current uncertainties. We find that the differences in past work were primarily due to differences in the equations of state. Even with these uncertainties, the study of explosions resulting from the collapse of white dwarfs, can help determine the viability of AICs as models for Type Ia supernovae and gamma-ray bursts. In addition, by calculating the nucleosynthetic yields of the neutron rich ejecta from AICs, we can limit the event rate (following the procedure described in $\S 2$ ). The upper limit of the AIC rate is given in Table 1 and, if we use the possibly more reliable Lattimer \& Swesty (1991) equation of state, is roughly $10^{-7}-10^{-5} \mathrm{yr}^{-1}$. To change these timescales appreciably, there must be large changes in the electron fraction of the ejecta which, although unlikely, can not be excluded at this time.

\subsection{Neutron Star Populations}

Our upper limit on the event rate somewhat constrains the role AICs play in producing neutron stars. The Type II supernova rate $\left(\sim 10^{-2} \mathrm{yr}^{-1}\right.$ : Cappellaro et al. 1998$)$ is $3-4$ orders of magnitude higher than our upper limit of the AIC rate, so neutron stars formed from AICs will not make up a large fraction of the total population of galactic neutron stars. However, neutron stars formed via accretion induced collapse may not receive the same large kicks observed in neutron stars formed in Type II supernovae, and hence may be prime candidates for globular cluster neutron star populations. Bailyn \& Grindlay (1990) estimate that the AIC rate must be $\sim 10^{-5}-10^{-5} \mathrm{yr}^{-1}$ to explain the globular cluster neutron star population, barely consistent with our upper limit on the AIC event rate. In addition, the event rate does not preclude AICs explaining any peculiar neutron star systems.

\subsection{Nucleosynthesis}

Because of their neutron rich ejecta, AICs are prime sites for r-process nucleosynthesis (Wheeler, Cowan, \& Hillebrandt 1998). However, although we can constrain the AIC event rate from the nucleosynthetic yield with our current models, predicting yields of specific isotopes remains beyond our grasp. To predict precisely the nucleosynthetic yields from AICs, the physical processes that cause the largest variations in the results (in particular, the equation of state and the effects of general relativity, and possibly, the characteristics of the progenitor) must be precisely known. 


\subsection{Gamma-Ray Bursts}

To achieve the high Lorentz factors required to drive a gamma-ray burst, a viable mechanism must have both large explosion energies $\left(\sim 10^{51}\right.$ ergs for isotropic explosions) and eject very little mass $\left(\lesssim 10^{-5} M_{\odot}\right)$. For our most-likely models, AICs eject 4 orders of magnitude too much mass with an order of magnitude too little energy. In agreement with Woosley \& Baron (1992), this clearly rules out those gamma-ray burst models relying upon neutrino/anti-neutrino annihilation (Paczynski 1986; Goodman 1986; Goodman, Dar, \& Nussinov 1987; Paczynski 1990; Dar et al. 1992). It also rules out all magnetically beamed models of AICs (Shaviv \& Dar 1995; Yi \& Blackman 1997,1998; Dai \& Lu 1998). In beamed models, the actual ejecta that effects the gamma-ray burst is limited to the ejecta swept up in the beam. In order for these beamed models to avoid ejecting too much mass, the beaming fraction must be smaller than $0.01 \%$ of the sky (hence sweeping up only $0.01 \%$ of the mass). However, with such a small beaming fraction, the AIC event rate must be greater than $10^{-3} \mathrm{yr}^{-1}$ for this mechanism to make up the majority of the observed gamma-ray bursts. This is an order of magnitude above our upper limit for the AIC event rate and it appears that AICs are simply unable to meet the observed requirements of gamma-ray bursts.

\subsection{Type Ia Supernovae}

On the other hand, AICs do not eject enough nickel to match most Type Ia supernova light curves. The amount of nickel ejecta is tantalizingly close to the properties of some peculiar Type Ia supernovae with very low nickel masses (e.g. SN 1991bg: Filippenko et al. 1992). AICs may be able to explain some peculiar Ias, a possibility that could be confirmed by either neutrino detections or the discovery of a neutron star formed in these Ias.

This paper has benefitted from the contributions of many people. We are grateful to R. Hix for making available his nuclear statistical equilibrium code, to D. Swesty for his nuclear equation of state, to D. Nadyozhin for his equation of state, to C. Wingate for his graphics software, and to S. Woosley for the progenitor star and many useful discussions. We would especially like to thank Edward Baron for providing access to his equation of state, insightful advice, and encouragement. The work of C.F. and W.B. was

partially supported by NSF grant AST 9206738 and from the Swiss National Science Foundation. The work of M. H. was supported by a director funded post-doctoral fellowship at Los Alamos National Laboratory.

\section{REFERENCES}

Arnett, W.D., \& Truran, J.W., 1970, ApJ, 160, 959

Bailyn, C.D. \& Grindlay, J.E. 1988, Nature, 336, 48

Bailyn, C.D. \& Grindlay, J.E. 1990, ApJ, 353, 159

Baron, E., Cooperstein, J., \& Kahana, S., 1985, Phys. Rev. Lett., 55, 126

Baron, E., Cooperstein, J., Kahana, S., \& Nomoto 1987, ApJ, 320, 304

Benz, W., Hills, J.G, \& Thielemann, F.-K., 1989, ApJ, 342, 986 
Benz, W. 1991, An introduction to Computational Methods in Hydrodynamics, in Late Stages of Stellar Evolution and Computational Methods in Astrophysical Hydrodynamics, ed. C.B. de Loore, (Berlin:Springer), p. 259

Blinnikov, S.I., Dunina-Barkovskaya, N.V., \& Nadyozhin, D.K., 1996, ApJS, 106, 171

Bowers, R.L. \& Wilson, J.R., 1982, ApJS, 50, 115

Canal, R., 1997, Thermonuclear Supernovae, NATO ASI series, 486, 257

Canal, R., Isern, J., \& Labay, J. 1990, ARA\&A, 28, 183

Chen, K., \& Leonard, P.J.T. 1993, ApJ, 411, L75

Chen, K., \& Ruderman, M. 1993, ApJ, 408, 179

Colgate, S.A., Petschek, A.G., \& Kriese, J.T., 1980, ApJ, 237, L81

Dai, Z.G., \& Lu, T., 1998, A\&A, 333, L87

Dar, A., Kozlovsky, B., Nussinov, S., \& Ramaty, R. 1992, ApJ, 388, 164

Ergma, E. 1993, A\&A, 273, L38

Filippenko, A.V., Richmond, M.W., Branch, D., Gaskell, C.M., Herbst, W., Ford, C.H., Treffers, R.R., Matheson, T., Ho, L.C., \& Dey, A., 1992 AJ 1041543

Fryer, C.L., Benz, W., \& Herant, M., 1996, ApJ, 460, 801

Goodman, J. 1986, ApJ, 308, L47

Goodman, J., Dar, A., \& Nussinov, S. 1987, ApJ, 314, L51

Hartmann, D., Woosley, S.E., \& El Eid, M.F. 1986, ApJ, 307, 414

Herant, M., Benz, W., Hix, W.R., Fryer, C.L., \& Colgate, S.A. 1994, ApJ, 435, 339

Hillebrandt, W., Nomoto, K., \& Wolff, R.G., 1984, A\&A, 133, 175

Hix, W.R., \& Thielemann, F.K. 1996, ApJ, 460, 869

Janka, H.T., $1991 \mathrm{PhD}$ thesis

King, A.R., \& Lasota, J.-P., 1991, ApJ, 378, 674

Kulkarni, S.R., Narayan, R., \& Romani, R.W., 1990, ApJ, 356, 174

Lattimer, J.M., \& Swesty, F.D. 1991, Nuc. Phys. A, 535,331

Levermore, C.D., \& Pomraning, G.C., 1981, ApJ, 248, 321

Li, X.-D., \& van den Heuvel, E.P.J., 1997, A\&A, 322, L9

Liebert, J., 1980, ARA\&A, 18, 363

Mayle, R. \& Wilson, J.R. 1988, ApJ, 334, 909 
Nomoto, K. \& Kondo, Y. 1991, ApJ, 367, L19

Paczynski, B., 1986, ApJ, 308, L43

Paczynski, B. 1990, ApJ, 363, 218

Qian, Y.-Z., Fuller, G.M., Mathews, G.J., Mayle, R.W., Wilson, J.R., \& Woosley, S.E., 1993, Phys. Rev. Let., 71,1965

Ray, A., \& Kluzniak, W. 1990, Nature, 344, 415

Ruderman, M. 1991, ApJ, 366, 261

Shapiro, S.L., \& Teukolsky, S.A., 1983, Black Holes, White Dwarfs, and Neutron Stars, (New York: Wiley \& Sons, Inc.), p. 169

Shaviv, N.J., \& Dar, A., 1995, ApJ, 447, 863

Swesty, F.D., Lattimer, J.M., \& Myra, E.S., 1994, ApJ, 425, 195

Takahashi, K., El Eid, M.F., \& Hillebrandt, W. 1977, A\&A, 67, 185

van den Heuvel, E.P.J. 1984, A\&A,5,209

Van Riper, K.A., 1979, ApJ, 232, 558

Wheeler, J.C., Cowan, J.J., Hillebrandt, W., 1998, ApJ, 493, L101

Woosley, S.E. \& Weaver, T.A. 1986, ARA\&A, 24, 205

Woosley, S.E. \& Baron, E. 1992, ApJ, 391, 228

Yi, I., \& Blackman, E.G., 1997, ApJ, 482, 383

Yi, I., \& Blackman, E.G., 1998, ApJ, 494, L163

Yungelson, L., \& Livio, M., 1998, ApJ, 497, 168 
Table 1. AIC Simulations

\begin{tabular}{llcccccc}
\hline \hline $\begin{array}{l}\text { Model } \\
\text { Num. }\end{array}$ & \multicolumn{1}{c}{ EOS $^{\mathrm{a}}$} & $\begin{array}{c}\text { Flux } \\
\text { Limiter }\end{array}$ & $\begin{array}{c}\nu^{\mathrm{c}} \\
\text { Source Term }\end{array}$ & $\mathrm{Y}_{\mathrm{e}}>0.45$ & $\begin{array}{c}\mathrm{M}_{\mathrm{ej}}\left(M_{\odot}\right) \\
0.40<\mathrm{Y}_{\mathrm{e}}<0.45\end{array}$ & $\begin{array}{c}\mathrm{Y}_{\mathrm{e}}<0.40 \\
\text { Rate } \\
(\mathrm{AIC} / \mathrm{Myr})\end{array}$ \\
\hline 1 & SL & Hetal & TEH & 0.09 & 0.04 & 0.07 & $2.9(12.9)$ \\
2 & SL-2D & Hetal & TEH & 0.07 & 0.01 & 0.09 & $2.2(10.1)$ \\
3 & SL-2D (rot) & Hetal & TEH & 0.07 & 0.01 & 0.11 & $1.8(8.2)$ \\
4 & SL & Hetal & WB & 0.10 & 0.02 & 0.08 & $2.5(11.3)$ \\
5 & SL-375 & Hetal & TEH & 0.08 & 0.03 & 0.09 & $2.2(10.1)$ \\
6 & SL-GR & Hetal & TEH & 0.02 & 0.04 & 0.22 & $0.91(4.1)$ \\
7 & SL-Adv. Burn & Hetal & TEH & 0.07 & 0.05 & 0.05 & $4.0(18.1)$ \\
8 & SL & BW & TEH & $\mathbf{0 . 0 5}$ & $\mathbf{0 . 0 6}$ & $\mathbf{0 . 0 2}$ & $\mathbf{1 0 . 0}(\mathbf{4 5 . 2})$ \\
9 & SL & BW & WB & 0.07 & 0.03 & 0.03 & $6.7(30.2)$ \\
10 & SL-Adv. Burn & BW & TEH & 0.04 & 0.06 & 0.005 & $15.1(181.0)$ \\
11 & SL-GR & BW & TEH & 0.01 & 0.01 & 0.26 & $0.77(3.5)$ \\
12 & SL & LP & TEH & $\mathbf{0 . 0 5}$ & $\mathbf{0 . 0 6}$ & $\mathbf{0 . 0 1}$ & $\mathbf{1 5 . 1}(\mathbf{9 0 . 5})$ \\
13 & SL & LP & WB & 0.07 & 0.05 & 0.02 & $10.0(45.2)$ \\
14 & SL-Adv. Burn & LP & TEH & 0.06 & 0.03 & 0.02 & $10.0(45.2)$ \\
15 & SL-No Burn & LP & TEH & 0.1 & 0.07 & 0.03 & $6.7(30.2)$ \\
16 & SL-No Burn & LP & WB & 0.08 & 0.05 & 0.03 & $6.7(30.2)$ \\
17 & SL-GR & LP & TEH & 0.01 & 0.01 & 0.25 & $0.80(3.6)$ \\
18 & BCK & Hetal & TEH & 0.04 & 0.04 & 0.0 & NA \\
19 & BCK & Hetal & WB & 0.03 & 0.03 & 0.0 & NA \\
20 & BCK & BW & TEH & 0.0 & 0.0 & 0.0 & NA \\
21 & BCK & BW & WB & 0.0 & 0.0 & 0.0 & NA \\
22 & BCK & BW & TEH & 0.0 & 0.0 & 0.0 & NA \\
23 & BCK & BW & WB & 0.0 & 0.0 & 0.0 & NA \\
24 & SL & none & none & 0.20 & 0.0 & 0.0 & $\infty$ \\
& & & & & & \\
\hline
\end{tabular}

${ }^{a}$ We use either the Swesty-Lattimer (SL) or Baron-Cooperstein-Kahana (BCK) equations of state

${ }^{\mathrm{b}}$ The following flux limiters are employed: Herant et al. (Hetal); Bowers-Wilson (BW); Levermore-Pomraning (LP).

${ }^{\mathrm{c}}$ We use the detailed electron/positron emission rates of Takahashi, El Eid \&Hillebrandt (TEH-1977) or the simplified rates used by WB.

d These rates are the maximum allowed assuming the entire production of these neutron rich materials come from AICs. The number in parantheses denotes the limit on the rate if the true $Y_{e}$ of the ejecta is $10 \%$ higher than our simulations predict.

eThe 2-D models are SPH simulations with $\sim 8000$ particles. The rotational simulation assumes a white dwarf spinning with a period of $2 \mathrm{~s}$.

${ }^{\mathrm{f}} K_{s}=375 \mathrm{MeV}$. In all other SL EOS simulations, $K_{s}=180 \mathrm{MeV}$.

g This run includes general relativistic effects.

${ }^{\mathrm{h}}$ Some runs include an additional burning network to be used prior to the onset of nuclear statistical equilibrium. In other runs, we have turned off even the NSE network to test the range of effects from nuclear burning.

${ }^{i}$ The most probable outcomes are bold-faced. 
Table 2. BCK EOS models ${ }^{\mathrm{a}}$

\begin{tabular}{|c|c|c|c|c|}
\hline$B C K \gamma$ & $W_{s}$ & $K_{0}^{s y m}$ & $x k z$ & Result \\
\hline 1.5 & 31.5 & 180 & 2.0 & $-b$ \\
\hline 1.5 & 31.5 & 375 & 2.0 & - \\
\hline 2.5 & 31.5 & 180 & 2.0 & - \\
\hline 2.5 & 31.5 & 375 & 2.0 & - \\
\hline 3.5 & 31.5 & 180 & 2.0 & - \\
\hline 3.5 & 31.5 & 375 & 2.0 & - \\
\hline 1.5 & 25.0 & 180 & 2.0 & - \\
\hline 1.5 & 25.0 & 375 & 2.0 & - \\
\hline 3.5 & 25.0 & 180 & 2.0 & - \\
\hline 3.5 & 25.0 & 375 & 2.0 & - \\
\hline 1.5 & 38.0 & 180 & 2.0 & - \\
\hline 1.5 & 38.0 & 375 & 2.0 & - \\
\hline 3.5 & 38.0 & 180 & 2.0 & - \\
\hline 3.5 & 38.0 & 375 & 2.0 & - \\
\hline 1.5 & 25.0 & 180 & 1.5 & - \\
\hline 1.5 & 25.0 & 375 & 1.5 & - \\
\hline 3.5 & 25.0 & 180 & 1.5 & - \\
\hline 3.5 & 25.0 & 375 & 1.5 & - \\
\hline 1.5 & 38.0 & 180 & 1.5 & - \\
\hline 1.5 & 38.0 & 375 & 1.5 & - \\
\hline 3.5 & 38.0 & 180 & 1.5 & - \\
\hline 3.5 & 38.0 & 375 & 1.5 & - \\
\hline 1.5 & 25.0 & 180 & 2.5 & $0.05 M_{\odot}$ ejected \\
\hline 1.5 & 25.0 & 375 & 2.5 & - \\
\hline 3.5 & 25.0 & 180 & 2.5 & $0.05 M_{\odot}$ ejected \\
\hline 3.5 & 25.0 & 375 & 2.5 & - \\
\hline 1.5 & 38.0 & 180 & 2.5 & - \\
\hline 1.5 & 38.0 & 375 & 2.5 & - \\
\hline 3.5 & 38.0 & 180 & 2.5 & - \\
\hline 3.5 & 38.0 & 375 & 2.5 & - \\
\hline 2.5 & 25.0 & 180 & 2.0 & - \\
\hline 2.5 & 25.0 & 375 & 2.0 & - \\
\hline 3.5 & 25.0 & 180 & 2.0 & - \\
\hline 3.5 & 25.0 & 375 & 2.0 & - \\
\hline 2.5 & 25.0 & 180 & 2.5 & $0.05 M_{\odot}$ ejected \\
\hline 2.5 & 25.0 & 375 & 2.5 & - \\
\hline 2.5 & 31.5 & 180 & 2.5 & - \\
\hline 2.5 & 31.5 & 375 & 2.5 & - \\
\hline
\end{tabular}

${ }^{\mathrm{a}}$ In all these models we use the Levermore-Pomraning flux limiter and the full neutrino source terms.

${ }^{b}$ Except where specifically noted, the end result for these simulations was no explosion. Of course, this does not exclude mass ejection from neutrino winds. 


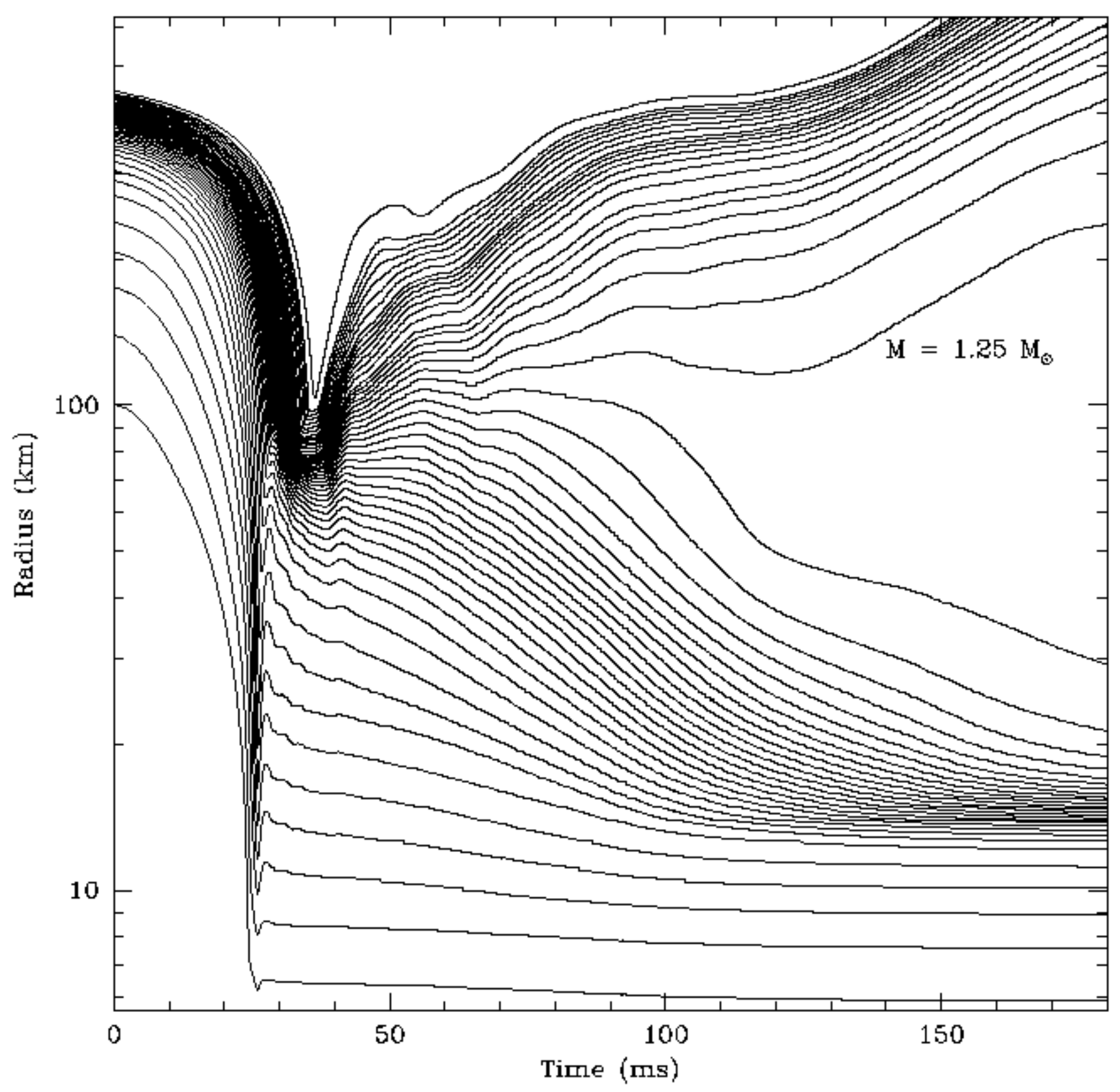

Fig. 1.- Mass-point trajectories for a simulation using the SL EOS. The resolution is increased near the transition between ejected material and proto-neutron star material. The inner 9 lines represent the $1 M_{\odot}$ core. 


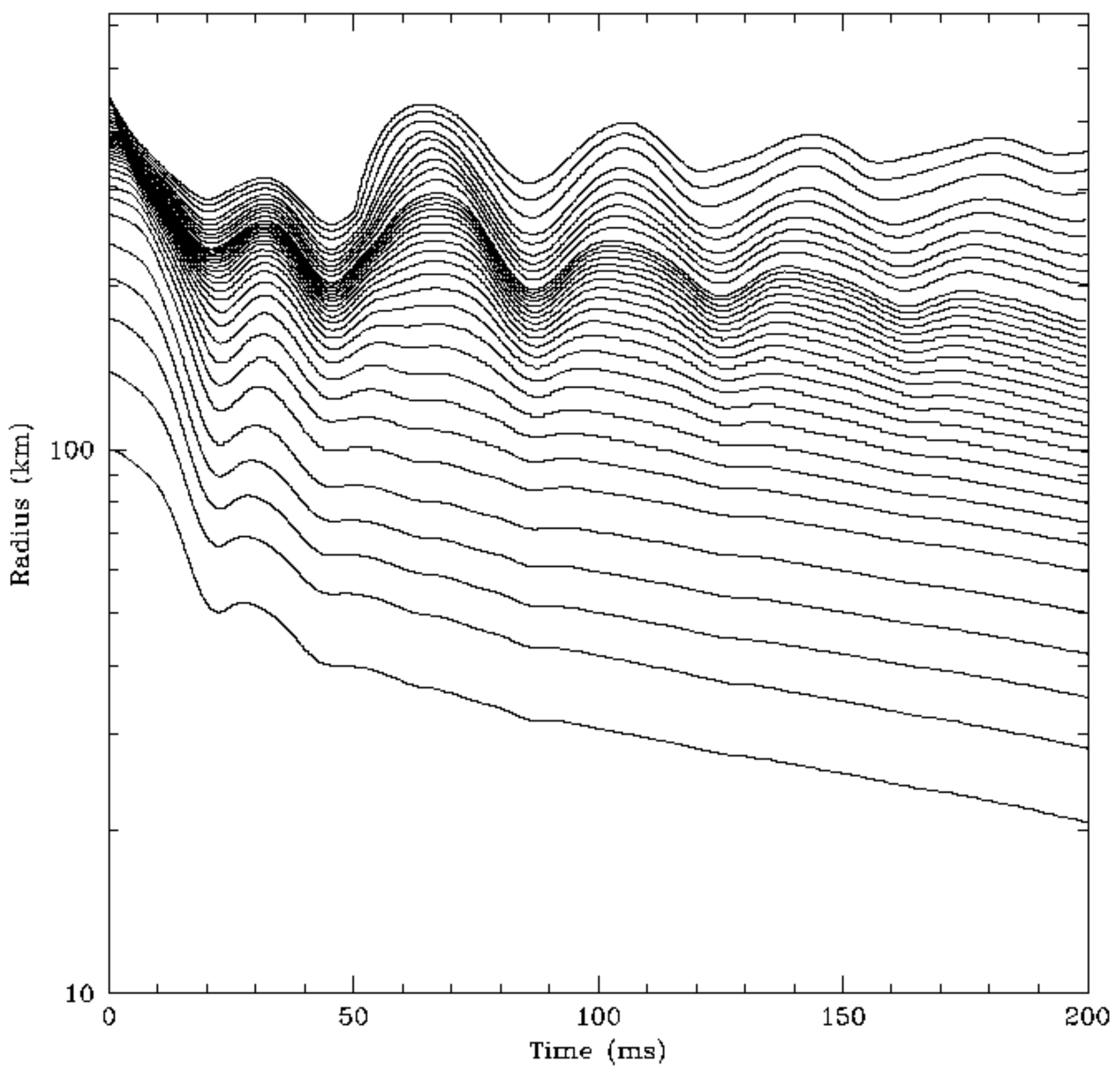

Fig. 2.- Identical to Figure 2 using the BCK equation of state. 


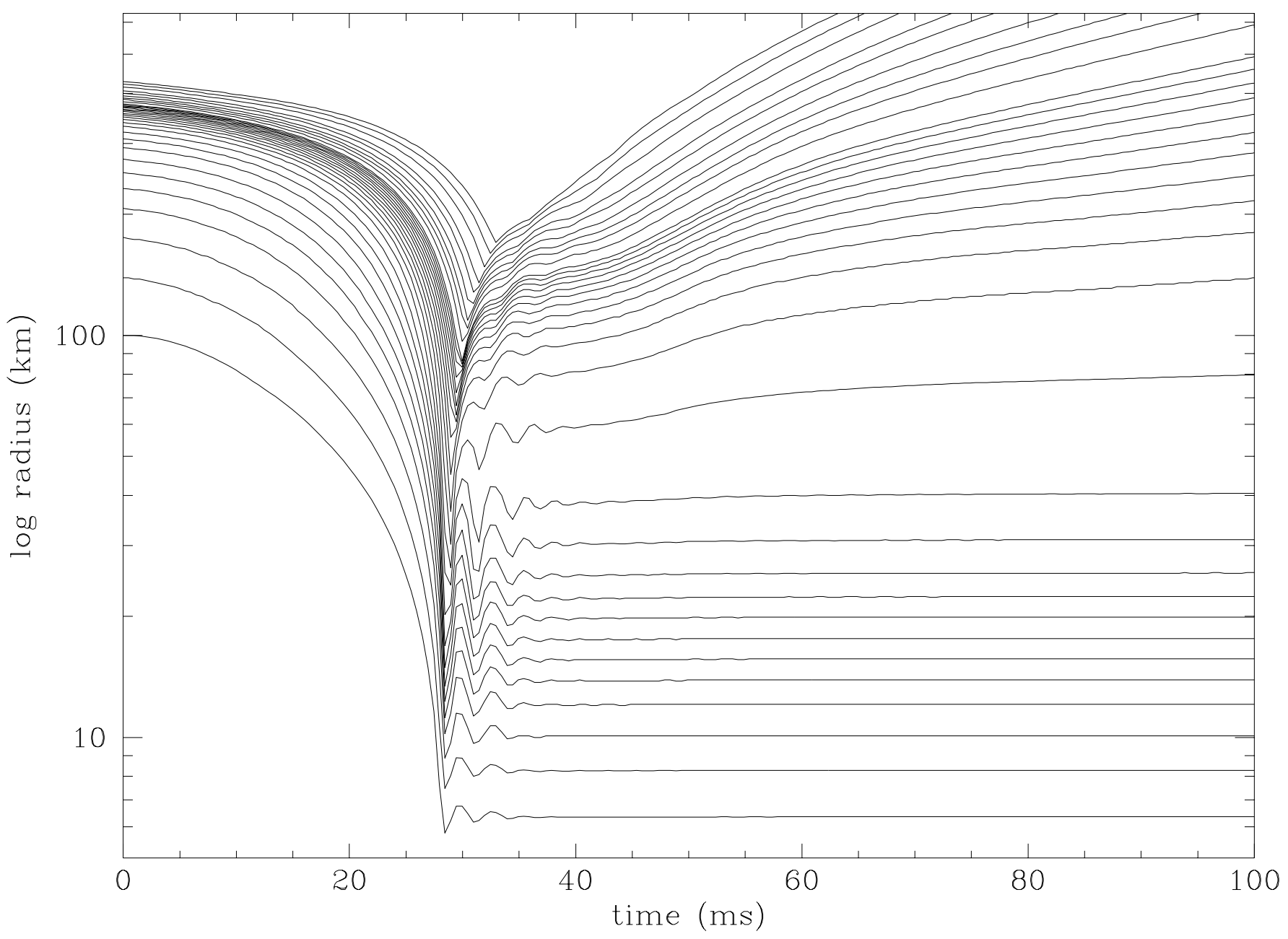

Fig. 3.- Identical to Figure 2 with the same SL EOS but without neutrino transport. Comparison with Figure 2 differentiates a prompt explosion from a delayed neutrino explosion. 


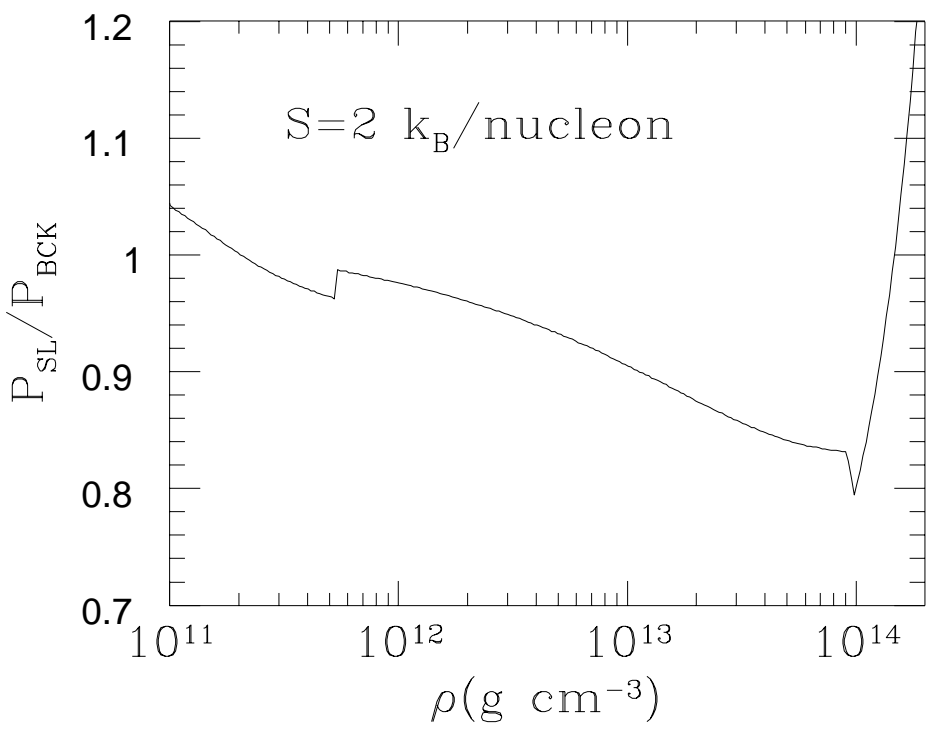

Fig. 4.- Ratio of pressure from the SL EOS and the BCK EOS. For densities less than $10^{14} \mathrm{~g} \mathrm{~cm}^{-3}$, the BCK pressure is higher by $10-20 \%$. Ye, KS 

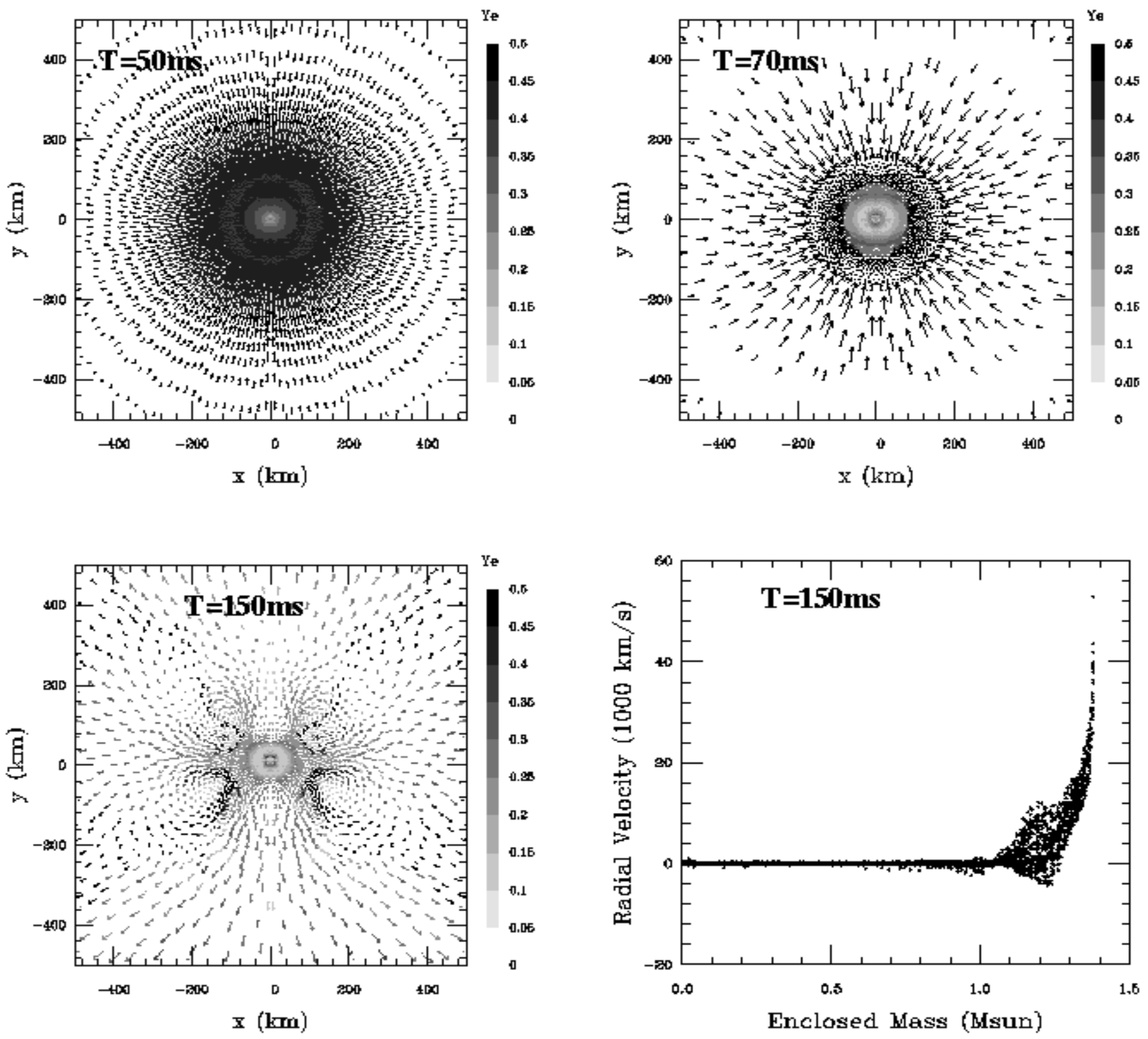

Fig. 5.- Time evolution of the collapse of a rotating $(20 \mathrm{~ms})$ white dwarf. At $50 \mathrm{~ms}$, the core of the white dwarf has already begun to rebound, but by $70 \mathrm{~ms}$, the shock has stalled and an accretion shock has formed. Note that the material falling along the equator (x-axis) is further out than the material infalling along the rotation axis (y-axis). At $150 \mathrm{~ms}$, the explosion has been launched. Although there is some convection, the explosion happens so quickly that it does not effect the ejecta significantly. In the lower right-hand corner, the radial velocity is plotted versus mass at $150 \mathrm{~ms}$. The outer $\sim 0.2 M_{\odot}$ is eventually ejected. The actual simulation is a $180^{\circ}$ wedge assuming cylindrical symmetry which have reflected about the vertical axis. 